\title{
miR-508-3p suppresses the development of ovarian carcinoma by targeting CCNA2 and MMP7
}

\author{
FEI GUO $^{1 *}$, KAI ZHANG ${ }^{1 *}$, MEIYUE LI ${ }^{1}$, LEI CUI $^{2}$, GUOYAN LIU $^{1}$, YE YAN $^{1}$, WENYAN TIAN ${ }^{1}$, \\ FEI TENG ${ }^{1}$, YANFANG ZHANG ${ }^{1}$, CHAO GAO $^{1}$, JINPING GAO ${ }^{1}$, YINGMEI WANG ${ }^{1}$ and FENGXIA XUE ${ }^{1}$ \\ ${ }^{1}$ Department of Gynecology and Obstetrics, Tianjin Medical University General Hospital, Tianjin 300052; \\ ${ }^{2}$ Department of Gynecology and Obstetrics, Tianjin First Central Hospital, Tianjin 300192, P.R. China
}

Received September 20,2019; Accepted April 9, 2020

DOI: 10.3892/ijo.2020.5055

\begin{abstract}
Ovarian cancer is the most lethal gynecological tumor, and the 5-year survival rate is only $\sim 40 \%$. The poor survival rate is due to cancer diagnosis at an advanced stage, when the tumor has metastasized. A better understanding of the molecular pathogenesis of tumor growth and metastasis is needed to improve patient prognosis. MicroRNAs (miRs) regulate carcinogenesis and development of cancers. However, the role of miR-508-3p in ovarian cancer remains largely unknown. Thus, the present study aimed to investigate the possible functions of miR-508-3p in the modulation of development of ovarian cancer. The results of the present study demonstrated that miR-508-3p mimics inhibited ovarian cancer cell proliferation, migration and invasion. Reporter gene assay results demonstrated that miR-508-3p suppressed cancer cell proliferation by directly targeting the 3'-untranslated region (UTR) of cyclin A2 (CCNA2) and suppressed migration and invasion by directly targeting the 3'-UTR of matrix metalloproteinase 7 (MMP7). In addition, high CCNA2 and MMP7 expression levels were associated with low miR-508-3p expression in ovarian cancer tissues. Furthermore, miR-508-3p and CCNA2 were independent predictors for overall survival in patients with ovarian cancer. To the best of our knowledge, this is the first study to demonstrated that miR-508-3p suppressed ovarian cancer development by directly targeting CCNA2 and $M M P 7$. The results of this study suggested the potential value of miR-508-3p and CCNA2 as prognostic indicators and therapeutics for ovarian cancer.
\end{abstract}

Correspondence to: Professor Fengxia Xue or Professor Yingmei Wang, Department of Gynecology and Obstetrics, Tianjin Medical University General Hospital, 154 Anshan Road, Heping, Tianjin 300052, P.R. China

E-mail: fengxiaxue1962@gmail.com

E-mail: wangyingmei1978@126.com

${ }^{*}$ Contributed equally

Key words: microRNA-508-3p, proliferation, metastasis, CCNA2, MMP7, ovarian carcinoma

\section{Introduction}

Ovarian cancer is the most lethal gynecological malignancy in the United States; in 2017, the estimated number of new cases for ovarian cancer was 22,440 , and the estimated number of deaths was 14,080 (1). In China, the estimated number of new cases of ovarian cancer was 52,100 , and the estimated number of deaths was 22,500 in 2015 (2). The high mortality of ovarian cancer is mainly due to its diagnosis at an advanced stage (3). Despite the progress in chemotherapy after surgery, most patients develop chemoresistance (4). Thus, 5-year survival is $\sim 40 \%$ for patients with ovarian cancer (5). An improved understanding of the genetic and molecular pathogenesis of this disease and targeted therapies are urgently needed to improve patient prognosis.

The human microRNA (miRNA/miR)-508-3p gene is located at Xq27.3, and alterations in miR-508-3p expression have been reported in several types of cancer (6-8). A previous study reported that low miR-508-3p expression levels are associated with an advanced stage of serous ovarian cancer (7), suggesting that deregulation of miR-508-3p in ovarian cancer may be associated with the tumorigenesis and progression of this disease. Zhai et al (6) reported that miR-508-3p suppressed cell proliferation and migration in renal cell carcinoma. miR-508-3p also exerts a tumor suppressor function by directly targeting nuclear factor- $\mathrm{\kappa B}$ subunit 1 and RELA proto-oncogene in gastric cancer (9). However, the role of miR-508-3p in ovarian cancer and the potential underlying molecular mechanisms remain largely unknown.

Cyclin A2 (CCNA2) regulates the cell cycle by binding and activating cyclin-dependent kinase 2 (CDK2) and thus promotes transition through the $\mathrm{G} 1 / \mathrm{S}$ and $\mathrm{G} 2 / \mathrm{M}$ phases. CCNA2 is overexpressed in various types of cancer, including ovarian cancer $(10,11)$. Matrix metalloproteinase 7 (MMP7) is a member of the MMP family and is also upregulated in multiple types of cancer, such as ovarian and colorectal cancer $(12,13)$. However, the molecular mechanisms leading to the upregulation of CCNA2 and MMP7 in ovarian cancer remain unclear. The present study aimed to examine the potential role of miR-508-3p in human ovarian cancer progression and to determine whether miR-508-3p inhibits cell proliferation, migration and invasion by directly targeting CCNA2 and MMP7 in ovarian cancer. 


\section{Materials and methods}

Patient samples. A total of 130 patients who were diagnosed with serous ovarian cancer at the Department of Gynecology and Obstetrics in Tianjin Medical University General Hospital (Tianjin, China) according to a pathological report between January 2009 and December 2016 were selected for the current study. The mean age of the patients was 57 years (range, 35-76 years). This study was approved by the Ethics Committee of Tianjin Medical University General Hospital. All participants signed consent forms prior to the surgical procedure. Pathological specimens were collected during the primary surgery. The ovaries of patients were resected during the surgery, and the adjacent non-tumor tissues were collected $1.0 \mathrm{~cm}$ away from the tumor. The specimens were fixed in formalin in room temperature for $24 \mathrm{~h}$ and embedded in paraffin. Then, 5- $\mu \mathrm{m}$ continuous sections were prepared for HE staining, in situ hybridization of miR-508-3p and immunohistochemical staining of CCNA2, MMP7 and proliferation marker protein Ki67. Each slide was re-evaluated by an expert pathologist without knowledge of patient clinical data before the experiments were performed. Only specimens containing $>70 \%$ of tumor tissue were used for the study. The cases were classified according to the FIGO stage system (2013) (14). The clinicopathological data were collected, including age, FIGO stage and grade (Table SI). In this cohort, the median follow-up duration was 36.8 months (range, 1.2-94 months). A total of $78 \%$ of patients were alive at the last follow-up. Overall survival (OS) times were calculated from the initial cytoreductive surgery and considered censored for patients who were alive at or died after the last follow-up.

To further evaluate the results of the survival data from Tianjin Medical University General Hospital, the ovarian carcinoma cohort was obtained from The Cancer Genome Atlas (TCGA; https://portal.gdc.cancer.gov/). The association between miR-508-3p expression and survival of 452 patients with ovarian cancer was analyzed using TCGA data.

Methods and cell culture. The ovarian cancer cell lines SKOV3, HeyA8 and A2780, and the cervical cancer cell line HeLa were obtained from the American Type Culture Collection and maintained in RPMI-1640 medium (Gibco; Thermo Fisher Scientific, Inc.) supplemented with 10\% FBS (Gibco; Thermo Fisher Scientific, Inc.) without any antibiotics. The cells were incubated at $37^{\circ} \mathrm{C}$ in an atmosphere containing $5 \% \mathrm{CO}_{2}$. Human miR-508-3p mimic (hsa-miR-508-3p) and scrambled negative control (miR-ctrl) were obtained from GE Healthcare Dharmacon, Inc. Small interfering RNA (siRNA) targeting CCNA2 or MMP7 and scrambled negative siRNA control were purchased from Sigma-Aldrich; Merck KGaA. The pcDNA3.1 plasmids (Promega Corporation) expressing CCNA2 or MMP7 and empty vectors (EV) were constructed by Shanghai GenePharma Co., Ltd.

miRNA, siRNA and plasmid transfection. The ovarian cancer cell lines SKOV3, HeyA8 and A2780 (2x10 $0^{5}$ per well) were seeded in 6-well plates and allowed to attach for at least 16 h. miR-508-3p mimic (miR-508-3p), miR-ctrl or siRNA were transfected into cells using Lipofectamine ${ }^{\circledR}$ RNAiMAX (Invitrogen; Thermo Fisher Scientific, Inc.) at a final concentration of $50 \mathrm{nM}$. Plasmids (500 ng) were transfected into cells using Lipofectamine ${ }^{\circledR} 2000$ (Invitrogen; Thermo Fisher Scientific, Inc.). The transfection reagents were used according to the manufacturer's instructions, and the transfections were performed at $37^{\circ} \mathrm{C}$ for $48 \mathrm{~h}$. Total RNA and protein were collected $48 \mathrm{~h}$ post-transfection. Cell supernatants were collected at $24 \mathrm{~h}$ for ELISA.

Reverse transcription-quantitative (RT-q) PCR. Total RNA were isolated from the ovarian cancer cell lines SKOV3, HeyA8 and A2780 using the mirVana ${ }^{\mathrm{TM}}$ miRNA Isolation kit (Ambion; Thermo Fisher Scientific, Inc.) according to the manufacturer's instructions. A total of $50 \mathrm{ng}$ RNA was reverse transcribed to cDNA using a Taqman MicroRNA Reverse Transcription kit (cat. no. 4366596; Applied Biosystems; Thermo Fisher Scientific, Inc.) for the reverse transcription of miRNA and $\mathrm{U} 6\left(16^{\circ} \mathrm{C}\right.$ for $30 \mathrm{~min}, 42^{\circ} \mathrm{C}$ for $30 \mathrm{~min}, 85^{\circ} \mathrm{C}$ for $5 \mathrm{~min}$ and store at $4^{\circ} \mathrm{C}$ ) or Taqman Reverse Transcription reagents (cat. no. 8080234; Applied Biosystems; Thermo Fisher Scientific, Inc.) for the reverse transcription of CCNA2 and MMP7 mRNA $\left(25^{\circ} \mathrm{C}\right.$ for $10 \mathrm{~min}, 42^{\circ} \mathrm{C}$ for $50 \mathrm{~min}, 70^{\circ} \mathrm{C}$ for $15 \mathrm{~min}$ and store at $4^{\circ} \mathrm{C}$ ). TaqMan RT-PCR assay probes for CCNA2 (cat. nos. A15629 and A15630) and MMP7 (cat. nos. A15629 and A15630) were purchased from Thermo Fisher Scientific, Inc. TaqMan miRNA assay kits were purchased from Applied Biosystems; Thermo Fisher Scientific, Inc (cat. no. 4427975).

The probes of PCR for miR-508-3p (cat. no. AP01571) and U6 (cat. no. AP01501) were purchased from HaiGene Co., Ltd. The qPCR instrument used was Applied Biosystems Real-Time PCR System (BioRad Laboratories, Inc.) with the following thermocycling conditions: $95^{\circ} \mathrm{C}$ for $10 \mathrm{~min}$, followed by 50 cycles of $95^{\circ} \mathrm{C}$ for $15 \mathrm{sec}$ and $60^{\circ} \mathrm{C}$ for $1 \mathrm{~min}$. U6 and GAPDH were used as normalization controls. Data were analyzed by the $2^{-\triangle \Delta \mathrm{Cq}}$ method (15).

MTT assay. The 3-(4,5-dimethyl-2-thiazolyl)-2,5-diphenyltetrazolium bromide (MTT) substrate (Sigma-Aldrich; Merck $\mathrm{KGaA}$ ) was used to assay cell proliferation according to the manufacturer's instructions. Briefly, SKOV3, HeyA8 and A2780 cells transfected with miR-508-3p, miR-ctrl, siRNA-ctrl, siRNA-CCNA2 or siRNA-MMP7 were seeded into 96-well plates at a density of $1 \times 10^{3}$ cells/well with $200 \mu \mathrm{l}$ RPMI-1640 medium supplemented with 10\% FBS. Then, $5 \mathrm{mg} / \mathrm{ml}$ MTT reagent was added to the medium (without FBS) at a ratio of $1: 9$ and was used to replace the medium in each well (100 $\mu \mathrm{l} /$ well) at different time points (1, 2, 3 and 4 days), followed by incubation at $37^{\circ} \mathrm{C}$ for $4 \mathrm{~h}$. After removal of the medium, $150 \mu$ l dimethylsulfoxide was added to each well, and the cells were gently agitated for $5 \mathrm{~min}$ at $37^{\circ} \mathrm{C}$. Absorbance values were determined using a microplate reader at $590 \mathrm{~nm}$. Each experiment contained three replicates.

Colony formation assay. The ovarian cancer cell lines SKOV3, HeyA8 and A2780 were harvested $24 \mathrm{~h}$ after transfection with miR-508-3p or miR-ctrl, seeded in a fresh 6-well plate (1,000 cells/well) and incubated for 14 days. Colony formation was analyzed by staining cells with $0.1 \%$ crystal violet at room temperature for $30 \mathrm{~min}$. The colonies were observed under an inverted phase contrast microscope (Olympus corporation), and a cluster containing $>50$ cells was considered a colony. The effi- 
ciency of colony formation was calculated as follows: Colony formation rate $=($ No. of colonies $/$ No. of seeded cells $) \times 100 \%$.

Cell cycle analysis. SKOV3, HeyA8 and A2780 cells were transfected with miR-508-3p, miR-ctrl, siRNA-ctrl or siRNA-CCNA2. At $48 \mathrm{~h}$ post-transfection, the cells were harvested by trypsinization, washed with PBS and fixed in ice-cold $70 \%$ ethanol at $4^{\circ} \mathrm{C}$ overnight. The cells were then washed in PBS and incubated with $50 \mu 1100 \mathrm{~g} / \mathrm{ml}$ ribonuclease (Thermo Fisher Scientific, Inc.) for $20 \mathrm{~min}$ at room temperature to ensure that only the DNA was stained. Then, the cells were incubated in $200 \mu 150 \mathrm{~g} / \mathrm{ml}$ propidium iodide for $30 \mathrm{~min}$ at $37^{\circ} \mathrm{C}$ in the dark. Cell cycle distribution was assayed using a FACSCalibur ${ }^{\mathrm{TM}}$ Flow Cytometer (BD Biosciences). The data were analyzed with the ModFit LT v3.3 software (BD Biosciences).

Transwell assays. For Transwell experiments, $24 \mathrm{~h}$ post-transfection, SKOV3 and HeyA8 cells $\left(5 \times 10^{4}\right.$ cells/well) were seeded into the upper wells of the Transwell chamber in RPMI-1640 medium without serum. The Transwell chamber was pre-coated with Matrigel (BD Biosciences) for $30 \mathrm{~min}$ at $37^{\circ} \mathrm{C}$ for the invasion assay or left uncoated for the migration assay. The cells were allowed to migrate or invade toward RPMI-1640 medium containing $10 \%$ FBS for $24 \mathrm{~h}$ at $37^{\circ} \mathrm{C}$. Then, the cells on the lower surface were fixed with $4 \%$ paraformaldehyde at room temperature for $30 \mathrm{~min}$ and stained with $0.1 \%$ crystal violet at room temperature for $15 \mathrm{~min}$. The cells on the upper surface were removed using a cotton swab. The migrated or invaded cells were counted in six randomly selected fields (x400 magnification) under a positive phase contrast microscope (Olympus Corporation).

Western blot analysis. SKOV3 or HeyA8 cells were solubilized in RIPA Lysis and Extraction Buffer (Thermo Fisher Scientific, Inc.) supplemented with Halt Protease Inhibitor Cocktail (Thermo Fisher Scientific, Inc.). Protein quantification was performed using BCA Protein Assay kit (Abcam). Equal amounts $(40 \mu \mathrm{g})$ of protein from each sample was subjected to SDS-PAGE on a $10 \%$ SDS-acrylamide gel and loaded onto a PVDF membrane. The membrane was blocked by $5 \%$ skimmed milk TBS + Tween-20 (1,000:1) buffer at room temperature for $1 \mathrm{~h}$ and probed with the following primary antibodies: CCNA2 (1:200; cat. no. sc-596; Santa Cruz Biotechnology, Inc.), MMP7 (1:200; cat. no. sc-80205; Santa Cruz Biotechnology, Inc.), $\beta$-actin (1:1,000; cat. no. sc-8432; Santa Cruz Biotechnology, Inc.), MMP2 (1:1,000; cat. no. ab37150; Abcam), pRb (1:1,000; cat. no. 9307; Cell signaling Technology, Inc.) CDK4 (1:1,000; cat. no. sc-53636; Santa Cruz Biotechnology, Inc.), CDK6 (1:1,000; cat. no. sc-53638; Santa Cruz Biotechnology, Inc.) and Ki67 (1:1,000; cat. no. AF0198; Cell Signaling Technology, Inc.) overnight at $4^{\circ} \mathrm{C}$. The membrane was washed three times by TBS + Tween-20 (1000:1) buffer at room temperature for $10 \mathrm{~min}$. The secondary antibodies included horseradish peroxidase (HRP)-conjugated goat anti-rabbit $\operatorname{IgG}(1: 4,000$; cat. no. ab205718; Abcam) and HRP-conjugated horse anti-mouse $\operatorname{IgG}$ (1:6,000; cat. no. 7076; Cell Signaling Technologies, Inc.). The secondary antibody incubation was performed at room temperature for $1 \mathrm{~h}$. The proteins were visualized using the
SuperSignal West Pico Chemiluminescent Substrate (Pierce; Thermo Fisher Scientific, Inc.).

ELISA. The concentration of MMP7 in the conditioned media from cultured cells was determined by a MMP7 ELISA kit (cat. no. PDMP700; R\&D Systems, Inc.) according to the manufacturer's instructions. Briefly, the collected conditioned media from SKOV3 or HeyA8 cells were added to a well coated with an MMP7 polyclonal antibody and immunosorbed by a biotinylated monoclonal anti-human MMP7 antibody at $37^{\circ} \mathrm{C}$ for $1 \mathrm{~h}$. The color development catalyzed by horseradish peroxidase was terminated with $2.5 \mathrm{~mol} / 1$ sulfuric acid. The protein concentration was determined by a microplate reader at $450 \mathrm{~nm}$.

Luciferase reporter assay. The 3'-untranslated regions (UTRs) of the CCNA2 and MMP7 genes, each of which contained one putative miR-508-3p binding site identified by TargetScan (http://www.targetscan.org/vert_71/) and miRBase (http://www.mirbase.org/) databases, were amplified by PCR from cDNA derived from HeyA8 cells and inserted into the multiple cloning sites of the pmirGLO vector (Promega Corporation). The primers used were as follows: CCNA2 forward, 5'-AAGTATATGGTGTACAGTTTTTAACTTAG GTTTTAATTTTTTCTGAATACAGAAGTTGTG-3' and reverse, 5'-CACAACTTCTGTATTCAGAAAAAATTAAA ACCTAAGTTAAAAACTGTACACCATATACTT-3'; MMP7 forward, 5'-CGGCTAGCTCAGGCAGAACATCCATT CATTC-3' and reverse, 5'-GCTCTAGACATTTATTGACA TCTACCCACTGCA-3'. Two mutant (mt) CCNA2 and MMP7 3'-UTR reporter vectors that lacked the binding sites for miR-508-3p were created through site-directed mutagenesis using a QuikChange kit (Stratagene; Agilent Technologies, Inc.). The primers used for site-directed mutagenesis were as follows: CCNA2 3'-UTR-mt forward, 5'-TACTTGTCAATAT TTAAACATGGTTTGCTGAAAATGGTATTTTCCCCC-3' and reverse, 5'-GGGGAAAATACCATTTTCAGCAAACC ATGTTTAAATATTGACAAGTA-3'; MMP7 3'-UTR-mt forward, 5'-GGATTGTATATCATTGTTGCGAATTGAT AAGCACTGTTCC-3' and reverse, 5'-GGAACAGTGCTTAT CAATTCGCAACAATGATATACAATCC-3'. All constructed clones were verified by DNA sequencing performed by Beijing Genomics Institute (Shenzhen, China).

For the luciferase reporter assay, $0.5 \mu \mathrm{g}$ pmirGLO, pmirGLO-3'-UTR-wild-type or pmirGLO-3'-UTR-M was transfected into HeLa cells that were cultured in 24-well plates together with $50 \mathrm{nM}$ miR-508-3p or miR-ctrl using Lipofectamine $^{\circledR} 2000$ (Invitrogen; Thermo Fisher Scientific, Inc.). At $48 \mathrm{~h}$ post-transfection, the cells were subjected to lysis, and firefly and Renilla luciferase activities were determined using a Dual-Luciferase Reporter Assay system (Promega Corporation) as previously described (16). Firefly luciferase activity was normalized to Renilla luciferase activity for each construct and compared with that of the miR-ctrl group.

miRNA in situ hybridization (ISH) and immunohistochemistry (IHC). miRNA ISH was performed as previously described (15). Briefly, the patient tissue sections were deparaffinized and incubated for $30 \mathrm{~min}$ at $37^{\circ} \mathrm{C}$ with $20 \mu \mathrm{g} / \mathrm{ml}$ proteinase $\mathrm{K}$. Then, the slides were dehydrated, followed by hybridization 

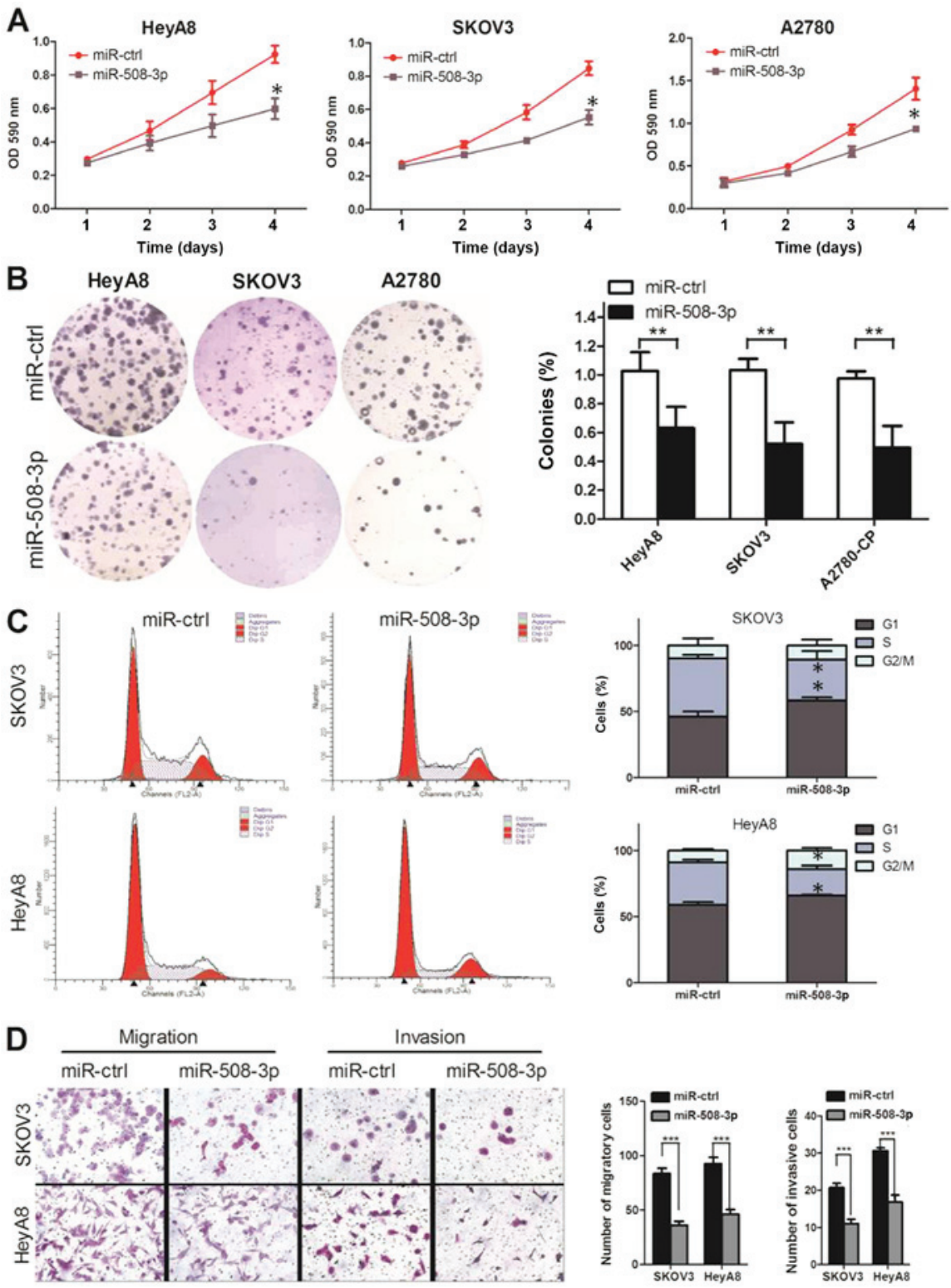

Figure 1. miR-508-3p functions as a tumor suppressor in ovarian cancer. (A) MTT assay results demonstrated that miR-508-3p inhibited the proliferation of HeyA8, SKOV3 and A2780 cells transfected with miR-508-3p or miR-ctrl. (B) Colony formation efficiency of the transfected ovarian cancer cells relative to the miR-ctrl groups. (C) miR-508-3p induced G1 phase arrest in ovarian cancer cells. The percentage of the miR-508-3p- or miR-ctrl-transfected ovarian cancer cells in each phase was assessed by flow cytometry. (D) Representative images and numbers of migratory or invasive cells following transfection with miR-508-3p or miR-ctrl analyzed by Transwell assays. Statistical comparisons were performed using the Wilcoxon test. ${ }^{*} \mathrm{P}<0.05,{ }^{* * *} \mathrm{P}<0.01,{ }^{* * * *} \mathrm{P}<0.001$ vs. miR-ctrl. miR, microRNA; ctrl, control.

with $100 \mathrm{nM}$ DIG-labeled miRCURY LNA ${ }^{\mathrm{TM}}$ detection probe hsa-miR-508-3p (cat. no. 611776-350; Qiagen $\mathrm{GmbH}$ ) for $2 \mathrm{~h}$ at $55^{\circ} \mathrm{C}$. Next, anti-DIG reagent (sheep anti-DIG-AP at 1:500 in antibody diluent; cat. no. 11093274910; Roche Applied Science) was added, and the sections were incubated for $60 \mathrm{~min}$ at room temperature. Then, freshly prepared alkaline phosphatase (AP) substrate [nitro blue tetrazolium chloride (NBT)/5-bromo-4-chloro-3-indolyl-phosphate, toluidine salt (BCIP); cat. no. 11697471001; Roche Applied Science) was applied to the sections, and the sections were incubated for $2 \mathrm{~h}$ at $30^{\circ} \mathrm{C}$ away from light. The U6 probe was used as a positive control for each section. The stained sections were reviewed by two independent pathologists under an inverted phase contrast microscope (Olympus Corporation) at x200 magnification. Signals in tumor cells were visually quantified using a scoring system between 0 and 9 ; the scores were assigned based on the intensity of the signal and the percentage of positive cells as follows: i) Signal: 0, no signal; 1, weak signal; 2, intermediate signal; and 3 , strong signal; and ii) percentage: $0,0 \% ; 1,<25 \%$; $2,25-50 \%$; and $3,>50 \%$. The scores were multiplied to achieve a final score. The low and high miR-508-3p expression groups were based on the mean of miR-508-3p ISH score and, the low and high CCNA2 or MMP7 expression groups were based on the mean of the IHC scores.

IHC staining was performed on the tumor tissues from 130 patients with serous ovarian cancer. The sections were immersed in eBioscience ${ }^{\mathrm{TM}}$ IHC Antigen Retrieval solution (cat. no. 00-4956-58; Thermo Fisher Scientific, Inc.) and heated in a microwave for $15 \mathrm{~min}$. Subsequently, the sections were blocked with goat serum (cat. no. 16210064; Thermo Fisher 

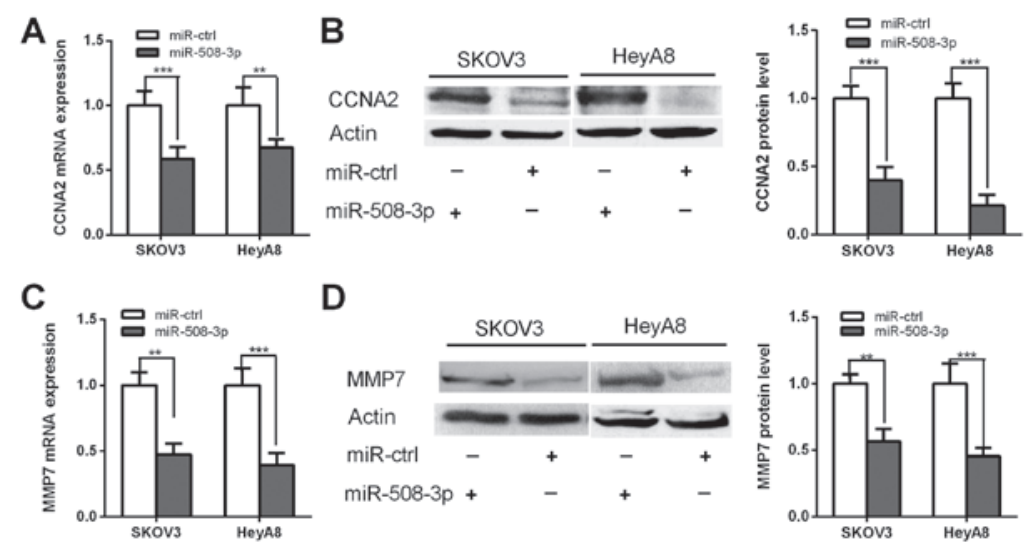

E

$$
\begin{aligned}
& \begin{array}{l}
\text { miR-508-3p 3' AGAUGAGGUUUUCCGAUGUUAGU } \\
\text { CCNA2 3'UTR }
\end{array} \\
& \text { nt 82-89 5' ...UUAGGUUUUAAUUUUACAAUCAU. } \\
& \text { CCNA2-mt 3'UTR 5'...UUAGGUUUUAAUUUAU. } \\
& \text { miR-508-3p 3' AGAUGAGGUUUUCCGAUGUUAGU } \\
& \text { MMP7 3'UTR IIIIII } \\
& \text { MMP7 3'UTR , AUAUUUAACAUGGUUACAAUCA } \\
& \begin{array}{cc}
\text { nt 56-62 } & \text { 5'...AUAUUUAAACAUGGUUACAA } \\
\text { MMP7-mt 3'UTR } & \text { 5'...AUAUUUAAACAUGGUU... }
\end{array}
\end{aligned}
$$

$\mathbf{F}$

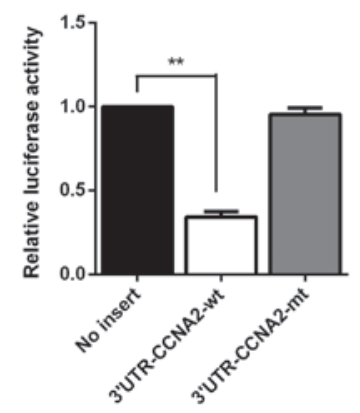

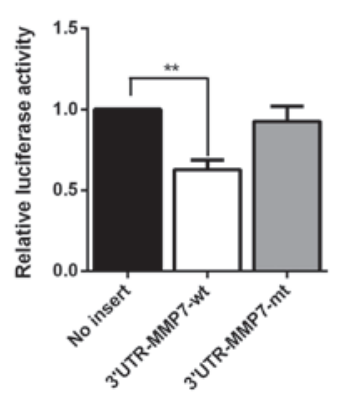

Figure 2. miR-508-3p directly targets CCNA2 and MMP7. Relative miRNA levels of (A) CCNA2 and (C) MMP7 in ovarian cancer cells transfected with miR-508-3p or miR-ctrl analyzed by reverse transcription-quantitative PCR and normalized to GAPDH. Protein levels of (B) CCNA2 and (D) MMP7 in ovarian cancer cells transfected with miR-508-3p or miR-ctrl analyzed by western blotting and normalized to $\beta$-actin. (E) miR-508-3p binding sites in the 3'-UTRs of CCNA2 and MMP7 were predicted by TargetScan. (F) Luciferase reporter assay in HeLa cells co-transfected with CCNA2- or MMP7 wt or mt 3'-UTR and miR-508-3p or miR-ctrl demonstrated that CCNA2 and MMP7 were direct targets of miR-508-3p. ${ }^{* * *} \mathrm{P}<0.01,{ }^{* * * *} \mathrm{P}<0.001$. miR, microRNA; ctrl, control; CCNA2, cyclin A2; MMP7, matrix metalloproteinase 7; UTR, untranslated region; wt, wild-type; mt, mutant.

Scientific, Inc.) at room temperature for $1 \mathrm{~h}$ and incubated with rabbit anti-human Ki-67 (1:200; cat. no. AP101M; EMD Millipore), mouse anti-human CCNA2 (1:200; cat. no. sc-596; Santa Cruz Biotechnology, Inc.) and mouse anti-human MMP7 (1:100, cat. no. sc-80205; Santa Cruz Biotechnology, Inc.) primary antibodies overnight at $4^{\circ} \mathrm{C}$. The secondary antibody (anti-rabbit IgG; cat. no. SPN-9001; OriGene Technologies, Inc.) was then added at room temperature for $1 \mathrm{~h}$. Finally, the sections were incubated with diaminobenzidine $(30 \mathrm{mg}$ dissolved in $100 \mathrm{ml}$ Tris buffer) for $5 \mathrm{~min}$, rinsed with running water and counterstained with hematoxylin for $1 \mathrm{~min}$ at room temperature.

Ki-67-positive cells were defined as those with brown staining in the nucleus, whereas MMP7-positive cells were defined as those with immunoreactivity in the cytoplasm. CCNA2-positive cells were considered as those with immunoreactivity in the nucleus and cytoplasm. The expression of these proteins was evaluated using the percentage of positive tumor cells in 1,000 tumor cells. The results were analyzed by two independent pathologists, and scores were assigned based on the intensity and the percentage of the stained tissues described above.
Statistical analysis. The results are presented as the mean \pm standard deviation. The cell culture experiments were performed at least three times and in triplicate. Wilcoxon test was performed using GraphPad Prism version 6 software (GraphPad Software, Inc.). Pearson's $\chi^{2}$ test, Kruskal-Wallis test with Dunn's post hoc test, Kaplan-Meier analysis, log-rank test and Cox's proportional hazards regression model were performed using SPSS 21.0 software (IBM Corp.). $\mathrm{P}<0.05$ was considered to indicate a statistically significant difference.

\section{Results}

miR-508-3p suppresses the proliferation, migration and invasion and increases the G1 phase distribution of ovarian cancer cells. miR-508-3p mimics were transfected into HeyA8, SKOV3 and A2780 cells. The results of the RT-qPCR analysis demonstrated increased expression of miR-508-3 compared with the miR-ctrl group in all the three cell lines $(\mathrm{P}<0.01$; Fig. S1), and the MTT assay revealed that miR-508-3p overexpression resulted in the suppression of cell proliferation at all time points compared with the miR-ctrl group (Fig. 1A). At $96 \mathrm{~h}$ post-transfection, miR-508-3p inhibited the viability of HeyA8 cells by 
A

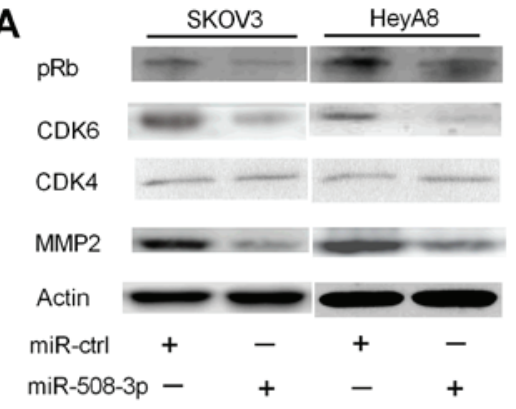

$\mathbf{B}=\begin{aligned} & \text { miR-ctrl } \\ & \text { miR-508-3p }\end{aligned}$

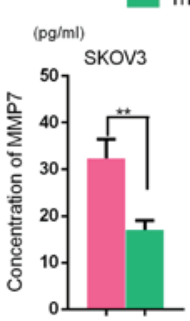

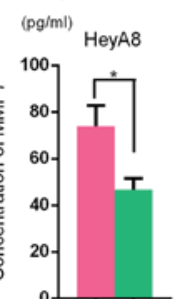
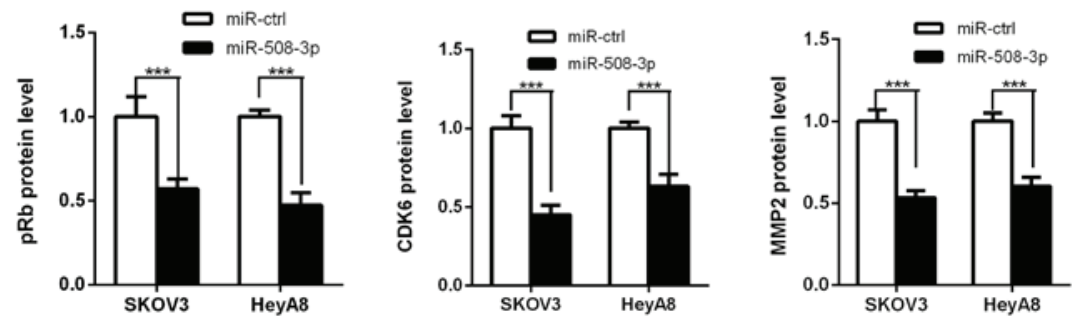

C

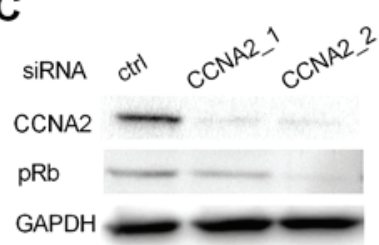

D
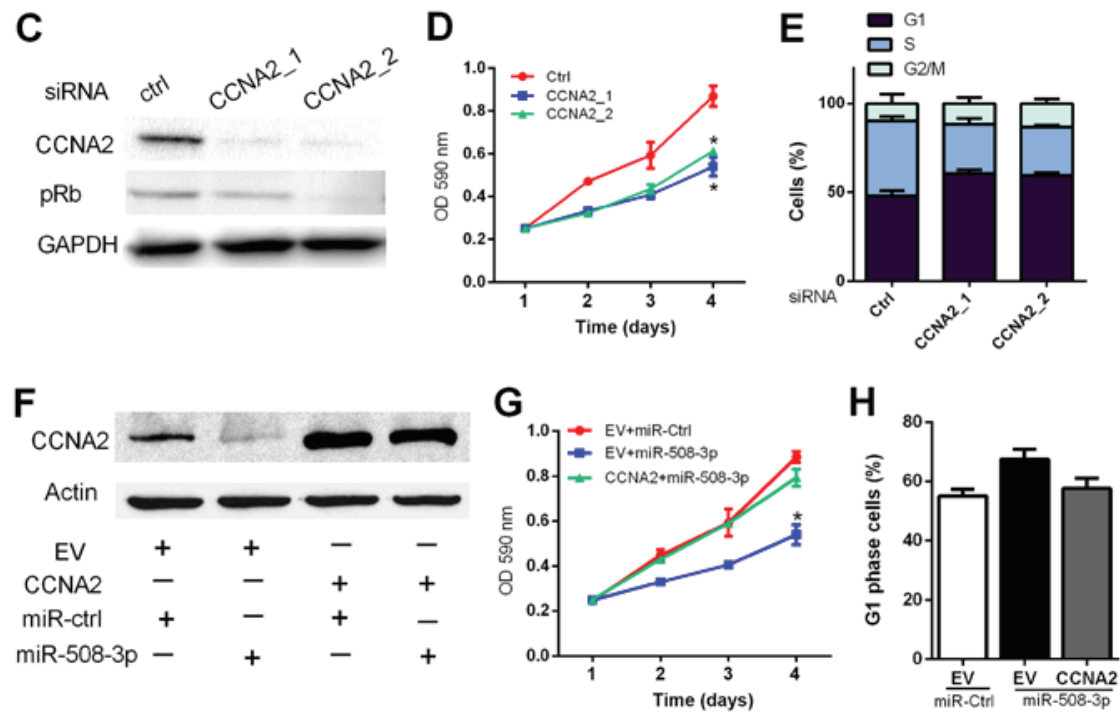

Figure 3. miR-508-3p suppresses CCNA2/pRb/CDK4/6 signaling and MMP7 levels, and CCNA2 is involved in miR-508-3p-induced inhibition of the cell cycle. (A) Western blot assay results demonstrated that miR-508-3p attenuated pRb, CDK6 and MMP2 protein levels. (B) Detection of MMP7 levels in the supernatant of ovarian cancer cells transfected with miR-508-3p or miR-ctrl by ELISA. (C-E) SKOV3 cells were transfected with siRNA-ctrl or siRNA-CCNA2, and the (C) protein level, (D) cell proliferation and (E) percentage of cells in each phase of the cell cycle were assessed by western blot, MTT and flow cytometry assays, respectively. (F-H) SKOV3 cells were co-transfected with CCNA2 vector or EV with miR-508-3p or miR-ctrl, and the (F) protein level, (G) cell proliferation and (E) percentage of cells in the G1 phase were assessed by western blot, MTT and flow cytometry assays, respectively. Kruskal-Wallis test was used to analyze the data. ${ }^{*} \mathrm{P}<0.05,{ }^{* *} \mathrm{P}<0.01,{ }^{* * *} \mathrm{P}<0.001$. miR, microRNA; ctrl, control; CCNA2, cyclin A2; MMP7, matrix metalloproteinase 7; siRNA, small interfering RNA; EV, empty vector.

$35.3 \%(\mathrm{P}<0.05)$, SKOV3 cells by $34.8 \%(\mathrm{P}<0.05)$ and $\mathrm{A} 2780$ cells by $33.4 \%(\mathrm{P}<0.05)$ compared with miR-ctrl (Fig. 1A). In addition, miR-508-3p impaired the colony formation efficiency of ovarian cancer cells; cells transfected with miR-508-3p displayed lower colony formation capacity compared with those transfected with miR-ctrl $(\mathrm{P}<0.05)$, further confirming the long-term anti-proliferative effect of miR-508-3p (Fig. 1B).

When miR-508-3p was transfected into HeyA8 and SKOV 3 cells, the proportion of cells in the $\mathrm{S}$ phase decreased compared with the corresponding miR-ctrl-transfected cells $(\mathrm{P}<0.05)$, and concomitantly, the proportion of cells in $\mathrm{G} 1$ phase significantly increased $(\mathrm{P}<0.05$; Fig. $1 \mathrm{C})$. To examine whether miR-508-3p was involved in apoptosis, Annexin $\mathrm{V}$ apoptosis assay was performed after transfection of miR-508-3p or miR-ctrl. SKOV3 and HeyA8 cells exhib- ited a mild non-significant increase in apoptotic rates at $48 \mathrm{~h}$ post-transfection with miR-508-3p compared with those in the miR-ctrl groups (data not shown). These results suggested that the suppressive effect of miR-508-3p on ovarian cancer cell proliferation was mediated by cell cycle arrest.

To examine the suppressive effects of miR-508-p on ovarian cancer cell invasion and migration, transient mimic transfections were performed, and the results demonstrated that miR-508-3p suppressed the migration and invasion of SKOV3 and HeyA 8 cells $(\mathrm{P}<0.05$; Fig. 1D). These results provided evidence that miR-508-3p may be an important inhibitor of the viability, migration and invasion of ovarian cancer cells.

miR-508-3p directly targets the 3'-UTRs of CCNA2 and $M M P 7$. To identify genes potentially regulated by miR-508-3p, 


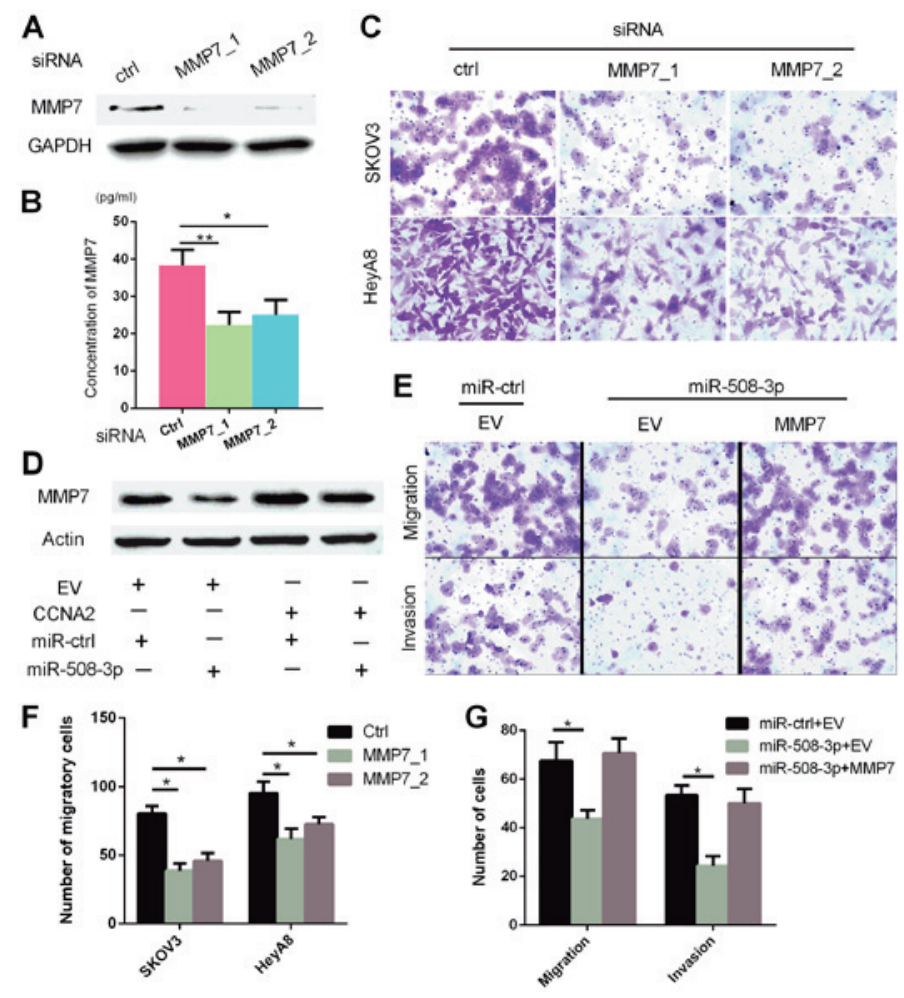

Figure 4. MMP7 is involved in the miR-508-3p-induced inhibition of ovarian cancer cell migration and invasion. (A-C) SKOV3 cells were transfected with siRNA-ctrl or siRNA-MMP7, and the (A) protein level of MMP7, (B) supernatant level of MMP7 and (C) migratory capacity were analyzed by western blot, ELISA and Transwell assays, respectively. (D-E) SKOV3 cells were co-transfected with the MMP7 vector or EV and miR-508-3p or miR-ctrl, and the (D) protein level and (E) invasive capacity were assessed by western blot and Transwell assays, respectively. Quantification results of (C and E) are presented in (F and G), respectively. Kruskal-Wallis test was used in this figure. "P $<0.05,{ }^{* *} \mathrm{P}<0.01$ vs. control. miR, microRNA; ctrl, control; CCNA2, cyclin A2; MMP7, matrix metalloproteinase 7; siRNA, small interfering RNA; EV, empty vector.

the TargetScan and miRBase databases were searched, and $C C N A 2$ and $M M P 7$ were identified as potential target genes of miR-508-3p. TargetScan analysis predicted one miR-508-3p binding site in the 3'-UTR of the CCNA2 gene and one in the 3'-UTR of the MMP7 gene (Fig. 2E), suggesting that miR-508-3p may directly target $C C N A 2$ and MMP7. To confirm this, SKOV3 and HeyA8 cells were transfected with miR-508-3p or miR-ctrl followed by measurement of CCNA2 and $M M P 7$ mRNA and protein levels $48 \mathrm{~h}$ post-transfection. The results demonstrated that $C C N A 2$ and $M M P 7$ mRNA (Fig. 2A and C) and protein (Fig. 2B and D) levels were significantly downregulated following miR-508-3p transfection compared with those in the miR-ctrl groups in SKOV3 and HeyA8 cells.

Luciferase reporter assays were performed to examine whether miR-508-3p directly targeted CCNA2 and MMP7. The 3'-UTRs of CCNA2 or MMP7 were cloned into the pmirGLO vector to generate pmirGLO-CCNA2 and pmirGLO-MMP7 constructs. Co-transfection of pmirGLO-CCNA2 with miR-508-3p into HeLa cells resulted in $65.7 \%$ lower level of luciferase activity compared with that following co-transfection with miR-ctrl, suggesting that miR-508-3p directly targeted CCNA2 (Fig. 2F). Similarly, co-transfection of pmirGLO-MMP7 with miR-508-3p resulted in $37.3 \%$ less luciferase activity compared with co-transfection with miR-ctrl, suggesting that miR-508-3p directly targeted $M M P 7$ (Fig. 2F).

To confirm that miR-508-3p specifically regulated $C C N A 2$ and MMP7 through the predicted binding sites, the constructs
pmirGLO-CCNA2-mt and pmirGLO-MMP7-mt, in which the miR-508-3p binding site sequences on the 3'-UTRs of the two genes were deleted, were generated and co-transfected with miR-508-3p or miR-ctrl into HeLa cells. Deletion of the miR-508-3p binding sites from the 3'-UTR of CCNA2 or $M M P 7$ abolished the effects of miR-508-3p on the luciferase activity (Fig. 2F). These results indicated that the CCNA2 and $M M P 7$ genes were direct targets of miR-508-3p.

miR-508-3p inhibits CCNA2/pRb/CDK4/6 signaling and decreases MMP7 levels in cells and culture supernatants. The possible mechanism of the suppressive effects of miR-508-3p on cancer cell proliferation may be induced by CCNA2/pRb/CDK4/6 (17); thus, the expression levels of pRb, CDK4 and CDK6 were determined in the present study. At $48 \mathrm{~h}$ post-transfection of miR-508-3p into SKOV3 and HeyA8 cells, the protein levels of $\mathrm{pRb}$ and CDK6 were decreased compared with those in cells transfected with miR-ctrl, but CDK4 levels did not change (Fig. 3A). These results indicated that miR-508-3p may suppress the proliferation of ovarian cancer cells by decreasing CCNA2, pRb and CDK6 levels.

Cell mobility may be induced by MMPs, which are known as direct regulators of tumor metastasis (18). In addition to MMP7, miR-508-3p also downregulated MMP2 protein levels (Fig. 3A). MMP7 and MMP2 levels in the culture supernatant were also examined, and the results demonstrated that the level of MMP7 was decreased after transfection of miR-508-3p compared with that in cells transfected with miR-ctrl (Fig. 3B), 
A

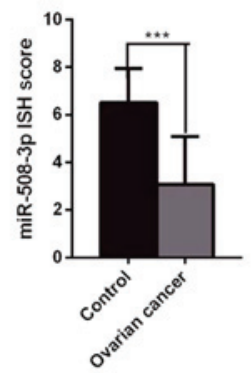

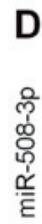
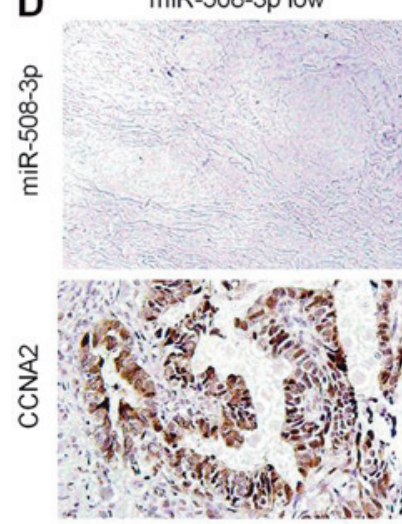

B

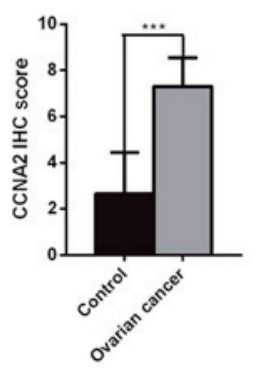

miR-508-3p high
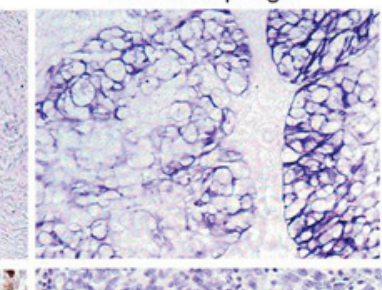

C

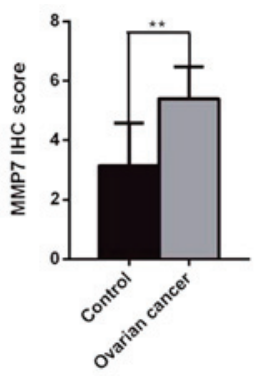

E
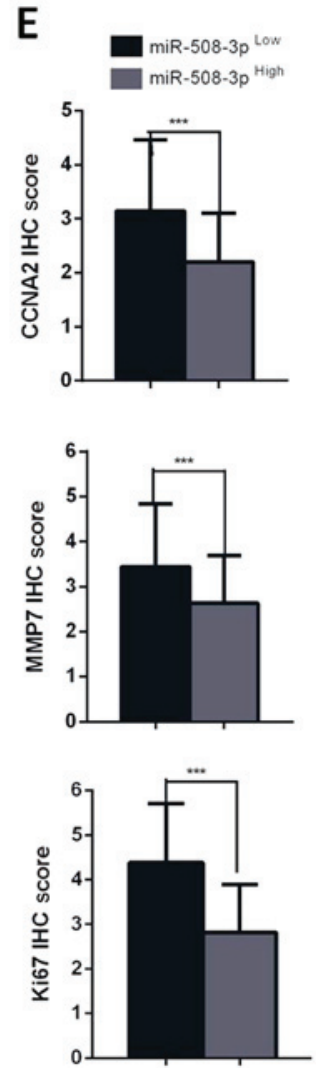

Figure 5. miR-508-3p expression in ovarian cancer is lower compared with that in the adjacent non-tumor tissue, and is associated with low CCNA2 and MMP7 expression levels. (A-C) Expression of (A) miR-508-3p, (B) CCNA2 and (C) MMP7 in ovarian cancer and adjacent non-tumor tissue. (D) Representative images of ISH of miR-508-3p and IHC for CCNA2, MMP7 and Ki67 in cases expressing low or high levels of miR-508-3p. (E) Bar charts demonstrating the association between the expression of miR-508-3p and CCNA2, MMP7 and Ki-67 determined by IHC. Student's t-test and log-rank test was used to analyze the data. Patients were stratified into high and low expression subgroups using the mean miR-508-3p, CCNA2 or MMP7 expression scores. ${ }^{* *} \mathrm{P}<0.01,{ }^{* * *} \mathrm{P}<0.001$. miR, microRNA; CCNA2, cyclin A2; MMP7, matrix metalloproteinase 7; ISH, in situ hybridization; IHC, immunohistochemistry.

indicating that miR-508-3p suppressed ovarian cancer cell migration and invasion by decreasing MMP7 levels.

CCNA2 is involved in the miR-508-3p-induced proliferation suppression and cell cycle arrest. To investigate whether the proliferation suppressive effect of miR-508-3p on ovarian cancer cells was mediated by repression of CCNA2, SKOV3 cells were transfected with siRNA-ctrl or siRNA-CCNA2. At $48 \mathrm{~h}$ post-transfection, knockdown of CCNA2 led to decreased expression of $\mathrm{pRb}$, mimicking the suppressive effect of miR-508-3p (Fig. 3C). At 4 days post-transfection, knockdown of CCNA2 led to a significant suppression of cell proliferation $(\mathrm{P}<0.05$; Fig. 3D). Cell proliferation was also suppressed at 2 and 3 days post-transfection, but no significant differences were observed between siRNA-CCNA2 and siRNA-ctrl when analyzed by the Kruskal-Wallis test $(\mathrm{P}>0.05$; Fig. 3D). Knockdown of CCNA2 also led to cell cycle arrest and increased G1 phase distribution, which was similar to the results of miR-508-3p overexpression; however, no statistical differences of G1 phase distribution were observed between siRNA-CCNA2 and siRNA-ctrl when analyzed by the Kruskal-Wallis test ( $\mathrm{P}>0.05$; Fig. 3E). These findings indicated that suppression of CCNA2 may be required for proliferation inhibition and cell cycle arrest.

Based on the above results, SKOV3 cells were co-transfected with miR-508-3p mimics and a plasmid overexpressing CCNA2 to further study whether ectopic expression of CCNA2 may rescue the inhibitory effects of miR-508-3p. As presented in Fig. 3F, overexpression of CCNA2 partially rescued the decreased expression of $\mathrm{pRb}$. At $48 \mathrm{~h}$ post-transfection, co-transfection of miR-508-3p and an empty vector led to significant suppression of cell proliferation compared with that of miR-ctrl and empty vector, which was attenuated by CCNA2 plasmid ( $\mathrm{P}<0.05$; Fig. 3G). Thus, ectopic expression of CCNA2 
Table I. Univariate log-rank analysis of prognostic factors in ovarian cancer.

\begin{tabular}{|c|c|c|c|c|c|}
\hline \multirow[b]{2}{*}{ Factors } & \multirow[b]{2}{*}{ No. (130) } & \multirow[b]{2}{*}{ No. dead } & \multicolumn{3}{|c|}{ Overall survival } \\
\hline & & & HR & $95 \% \mathrm{CI}$ & P-value \\
\hline \multicolumn{6}{|l|}{ Age, years } \\
\hline$<59$ & 65 & 12 & 2.265 & $1.069-4.797$ & $0.033^{\mathrm{a}}$ \\
\hline$\geq 59$ & 65 & 17 & & & \\
\hline \multicolumn{6}{|l|}{ Grade $^{b}$} \\
\hline Low & 33 & 5 & 1.411 & $0.5936-3.354$ & 0.436 \\
\hline High & 97 & 24 & & & \\
\hline \multicolumn{6}{|c|}{ FIGO stage ${ }^{b}$} \\
\hline $\mathrm{I}+\mathrm{II}$ & 40 & 6 & 2.194 & $1.025-4.696$ & $0.043^{\mathrm{a}}$ \\
\hline III+IV & 90 & 23 & & & \\
\hline \multicolumn{6}{|c|}{ Residual disease, $\mathrm{cm}$} \\
\hline$\leq 1$ & 95 & 13 & 4.294 & $2.792-15.16$ & $<0.001^{\mathrm{a}}$ \\
\hline$>1$ & 35 & 16 & & & \\
\hline \multicolumn{6}{|c|}{ Ascites cytology } \\
\hline Negative & 84 & 17 & 1.988 & $0.8722-4.530$ & 0.102 \\
\hline Positive & 46 & 12 & & & \\
\hline \multicolumn{6}{|c|}{ miR-508-3p } \\
\hline Low & 61 & 16 & 0.3224 & $0.1437-0.7234$ & $0.006^{\mathrm{a}}$ \\
\hline High & 69 & 13 & & & \\
\hline \multicolumn{6}{|l|}{ CCNA2 } \\
\hline Low & 81 & 18 & 2.390 & $1.269-7.739$ & $0.016^{\mathrm{a}}$ \\
\hline High & 49 & 11 & & & \\
\hline \multicolumn{6}{|l|}{ MMP7 } \\
\hline Low & 66 & 14 & 2.086 & $1.117-5.196$ & $0.033^{\mathrm{a}}$ \\
\hline High & 64 & 15 & & & \\
\hline
\end{tabular}

${ }^{a} \mathrm{P}<0.05$. ${ }^{\mathrm{b}} \mathrm{FIGO}$ staging system (2013). CI, confidence interval; miR, microRNA; CCNA2, cyclin A2; MMP7, matrix metalloproteinase 7.

partially rescued cell proliferation suppression mediated by miR-508-3p. In addition, co-transfection of miR-508-3p and an empty vector induced G1 phase arrest compared with that of miR-Ctrl and empty vector, but the difference was not statistically significant $(\mathrm{P}=0.05$; Fig. $3 \mathrm{H})$; this was also attenuated by CCNA2 plasmid. Taken together, these findings indicated that miR-508-3p may induce the proliferation suppression and cell cycle arrest of ovarian cancer cells by targeting CCNA2.

MMP7 is involved in the miR-508-3p-induced suppression of migration and invasion. To elucidate the possible mechanism by which miR-508-3p suppressed ovarian cancer cell migration and invasion, SKOV3 cells were transfected with siRNA-ctrl or siRNA-MMP7. At $48 \mathrm{~h}$ post-transfection with siRNA-MMP7, the MMP7 levels in the whole cell lysate and cultured supernatant were decreased compared with those in the siRNA-ctrl transfected cells $(\mathrm{P}<0.05$; Fig. 4A and B). In addition, SKOV3 and HeyA8 cells transfected with siRNA-MMP7_1 or siRNA-MMP7_2 exhibited significantly decreased migratory capacity compared with those transfected with siRNA-ctrl, which was in accordance with the effect of miR-508-3p ( $\mathrm{P}<0.05$; Fig. 4C and F).
To study whether ectopic expression of MMP7 may rescue the inhibition of migration and invasion, cells were co-transfected SKOV3 with miR-508-3p mimics and the plasmid expressing MMP7. As demonstrated in Fig. 4D and E, the suppressive effects of miR-508-3p were partially reversed by ectopic expression of MMP7. At $48 \mathrm{~h}$ post-transfection, miR-508-3p and empty vector led to a significant suppression of migration $(\mathrm{P}<0.05$; Fig. $4 \mathrm{E}$ and $\mathrm{G})$ and invasion $(\mathrm{P}<0.05$; Fig. 4E and G) compared with that in the miR-Ctrl and empty vector group, which was attenuated by the MMP7 plasmid. These results demonstrated that the effect of the miR-508-3p mimics was consistent with that of siRNA-MMP7 on SKOV3 cells, suggesting that miR-508-3p may inhibit the migration and invasion of ovarian cancer cells by directly targeting MMP7.

High miR-508-3p expression is associated with low CCNA2 and MMP7 expression and improved OS. Considering that miR-508-3p expression impaired tumor aggressiveness in the cellular models, its expression was evaluated in a cohort of 130 clinically annotated serous tumor samples. Expression of miR-508-3p and the control U6 small RNA was evaluated by 
Table II. Multivariate Cox regression analysis for overall survival in ovarian cancer.

Overall survival

\begin{tabular}{llll}
\cline { 2 - 4 } Factors & HR & 95\% CI & P-value \\
\hline Age (<59 vs. $\geq 59$ years) & 2.410 & $1.104-5.263$ & $0.027^{\mathrm{a}}$ \\
FIGO stage (I+II vs. III+IV) & 1.468 & $0.532-4.049$ & 0.458 \\
Residual disease ( $\leq 1$ vs. $>1 \mathrm{~cm})$ & 3.342 & $1.474-7.576$ & $0.004^{\mathrm{a}}$ \\
miR-508-3p (low vs. high) & 0.412 & $0.183-0.928$ & $0.032^{\mathrm{a}}$ \\
CCNA2 (low vs. high) & 2.429 & $1.025-5.756$ & $0.044^{\mathrm{a}}$ \\
MMP7 (low vs. high) & 2.084 & $0.881-4.929$ & 0.094 \\
\hline
\end{tabular}

${ }^{\mathrm{a} P}<0.05 .{ }^{\mathrm{b}} \mathrm{FIGO}$ staging system (2013). HR, hazard ratio; CI, confidence interval; miR, microRNA; CCNA2, cyclin A2; MMP7, matrix metalloproteinase 7.
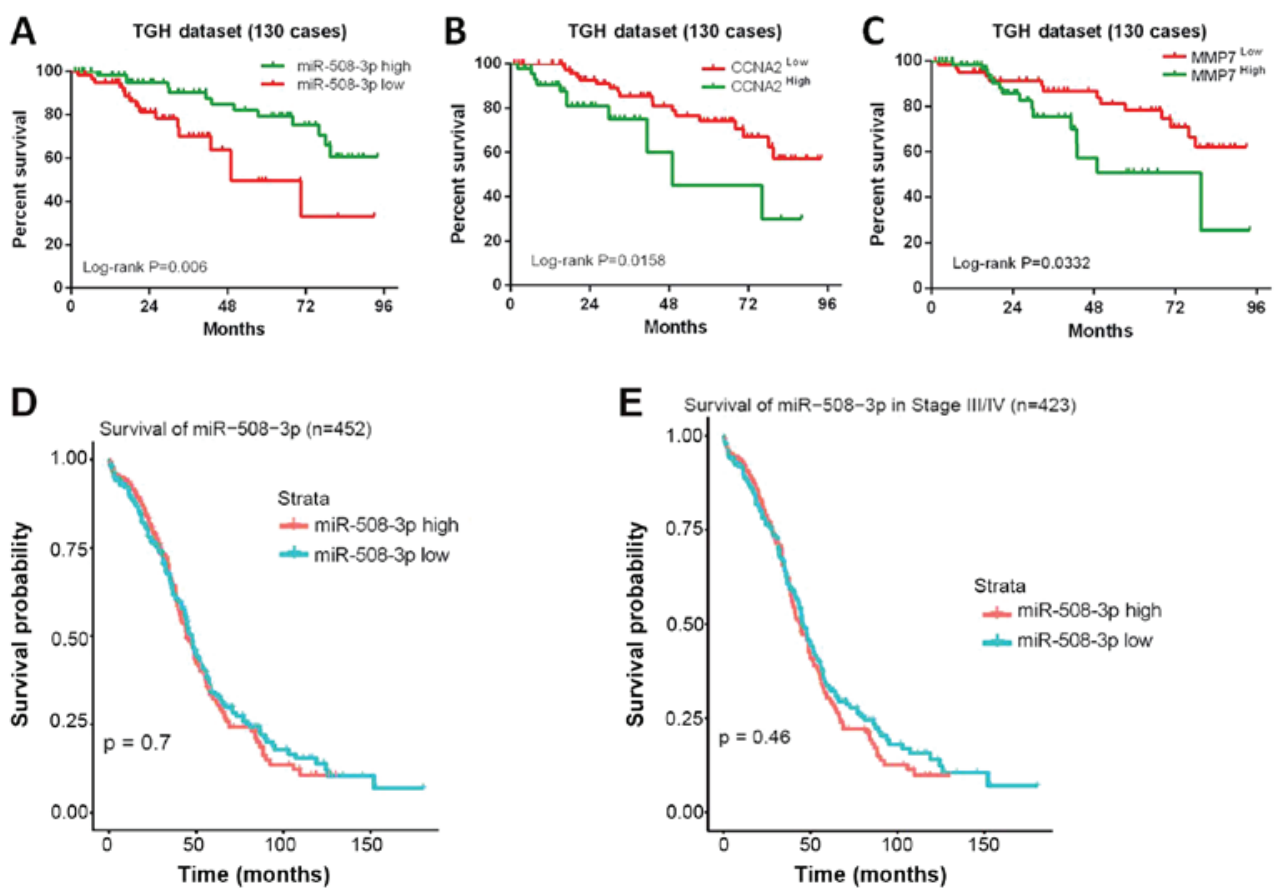

Figure 6. miR-508-3p expression is associated with longer overall survival of ovarian cancer. (A-C) Kaplan-Meier analysis of the association between (A) miR-508-3p, (B) CCNA2 or (C) MMP7 and the overall survival of all patients with ovarian cancer from Tianjin Medical University General Hospital. (D and E) Kaplan-Meier analysis of the association between miR-508-3p levels and the overall survival of (D) all patients with ovarian cancer and (E) patients with stage III-IV ovarian cancer from The Cancer Genome Atlas. miR, microRNA; CCNA2, cyclin A2; MMP7, matrix metalloproteinase 7.

ISH, whereas CCNA2 and MMP7 were evaluated by IHC in the same tissue samples.

To compare the levels of miR-508-3p, as well as CCNA2 and MMP7 in ovarian cancer with normal ovarian tissue, their expression was also evaluated in adjacent non-tumor tissue. The results demonstrated that miR-508-3p expression in ovarian cancer was significantly lower compared with that in adjacent non-tumor tissue $(\mathrm{P}<0.001$; Fig. 5A). By contrast, CCNA2 expression in cancer tissue was significantly higher compared with that in adjacent non-tumor tissue $(\mathrm{P}<0.001$; Fig. 5B). The results of MMP7 analysis were similar to those of CCNA2 ( $\mathrm{P}<0.05$; Fig. $5 \mathrm{C})$.

A significant decrease in miR-508-3p expression was observed with disease progression from stages I-II to III-IV ( $\mathrm{P}=0.028$; Table SI). No significant differences in clinicopathologic characteristics were observed between the CCNA2 low and high groups (Table SII) or between the MMP7 low and high groups (Table SIII). In addition, both CCNA2 and MMP7 IHC scores in the tissues from the miR-508-3p low group were significantly higher compared with those from the miR-508-3p high group $(\mathrm{P}<0.001$; Fig. 5D and 5E). The Ki67 expression score in the miR-508-3p low group was also significantly higher compared with that in the miR-508-3p high group $(\mathrm{P}<0.001)$. Univariate log-rank analysis demonstrated that older age, advanced FIGO stage, residual disease $>1 \mathrm{~cm}$, high CCNA2 and MMP7 and low miR-508-3p expression were significantly associated with a shorter OS time (Table I). A multivariate model revealed that age, residual disease size, miR-508-3p and CCNA2 were independent predictors for OS in ovarian cancer (Table II). 
Kaplan-Meier analysis demonstrated that patients with high expression levels of miR-508-3p exhibited longer OS times compared with those with low levels of miR-508-3p (median OS 43.91 vs. 27.90 months, respectively; log-rank $\mathrm{P}=0.006$; Fig. 6A). Accordingly, high levels of CCNA2 or MMP7 predicted a shorter OS time compared with low expression levels in a univariate analysis of patients with ovarian cancer (median OS 45.36 vs. 22.61 months, respectively; log-rank $\mathrm{P}=0.0158$ for CCNA2; median OS 45.15 vs. 28.16 months, respectively; log-rank $\mathrm{P}=0.0332$ for $\mathrm{MMP}$; Fig. 6B and $\mathrm{C}$ ).

To evaluate these results, ovarian carcinoma data from TCGA were analyzed. Among 452 patients with ovarian cancer, Kaplan-Meier analysis demonstrated that compared with patients with low miR-508-3p expression, those with high expression levels of miR-508-3p did not exhibit a longer OS time (log-rank $\mathrm{P}=0.07$; Fig. 6D). The same conclusion was reached for the 423 patients with stage III-IV ovarian cancer (log-rank $\mathrm{P}=0.46$; Fig. $6 \mathrm{E})$.

\section{Discussion}

Emerging evidence from previous studies has demonstrated that miRNAs regulate carcinogenesis and cancer development (19-21). Several studies have reported that miR-508-3p may function as a tumor suppressor or promoter in different tumors, such as renal cell carcinoma and gastric cancer $(6,9,22)$. But its exact roles and clinical relevance in ovarian cancer remain unclear. The results of the present study demonstrated that miR-508-3p may act as a tumor suppressor to inhibit cell proliferation, migration and invasion in ovarian cancer. To the best of our knowledge, the present study was the first to identify that CCNA2 and MMP7 were direct targets of miR-508-3p in ovarian cancer. In addition, the results demonstrated that the expression levels of miR-508-3p and CCNA2 were negatively associated, and that they were independent predictors for the OS time of patients with ovarian cancer.

The results of the present study demonstrated that miR-508-3p regulated the proliferation and cell cycle by directly binding to the 3 '-UTR of CCNA2 in ovarian cancer cells. In these experiments, HeLa cells were used for the Luciferase assays, as HeLa cells exhibit higher transfection efficiency compared with ovarian cancer cells. The results identified a direct binding site between miR-508-3p and the 3'-UTRs of the target genes. These direct targeted relationship is also likely to exist in ovarian cancer cells. CCNA2 is a main mitotic cyclin that controls the cell cycle by binding CDK1 and CDK2 (23). CCNA2 contributes to controlling the $\mathrm{S}$ phase by initiating DNA synthesis and, subsequently, mitotic entry (24). Abnormal expression of CCNA2 has been detected in various types of cancer, such as non-small cell lung cancer and colorectal carcinoma (25-27), and deregulation of CCNA2 is associated with chromosomal instability and tumor proliferation (28). CCNA2/CDK complexes phosphorylate proteins such as $\mathrm{Rb}(28)$, and phosphorylation of $\mathrm{RB}$ results in its inactivation and upregulation of CDK4/6, which allows progression through G1 into the S phase (29). The present results revealed that miR-508-3p induced proliferation suppression and G1 phase arrest in ovarian cancer cells, which were mediated by targeting the CCNA2/pRb/CDK4/6 signaling pathway. Thus, repressing CCNA2/pRb/CDK4/6 signaling may provide a rationale for the treatment of ovarian cancer.

To investigate the possible mechanisms that underlie the regulation of ovarian cancer cell migration and invasion potential by miR-508-3p, MMPs, which are known to be direct regulators of tumor metastasis, were examined in the present study. The results demonstrated that MMP2 and MMP7 were downregulated by miR-508-3p. MMP2 may not be directly regulated by miR-508-3p, as no miR-508-3p targeting sequence was identified in the 3'-UTR of MMP. Upregulation of MMP7, including in serum, has been reported in various types of cancer (30-32), including ovarian (12) and gastric cancer (30). In addition, high serum MMP7 levels alone (33) or in combinations with chromogranin A are independently associated with disease-specific survival in prostate cancer (34). MMP7 induces epithelial-to-mesenchymal transformation (EMT) in cancer by disrupting the E-cadherin $/ \beta$-catenin complex and upregulating EMT transcription factors (35), thus facilitating migration and invasion. The results of the present study demonstrated that MMP7 was a direct functional target of miR-508-3p via the analysis of the luciferase reporter assay. miR-508-3p downregulated the mRNA and protein levels of MMP7 in ovarian cancer cells. The results also confirmed that the miR-508-3p-induced attenuation of MMP7 levels, including those in the supernatant, significantly decreased the migration and invasion of ovarian cancer cells. These results were further validated by the rescue experiments.

The results of the present study indicated that miR-508-3p expression was decreased in serous ovarian cancer compared with that in normal ovarian tissue. A previous study has demonstrated that the methylation of miRNA promoters may explain the deregulation of miRNAs in cancer (15). However, the molecular mechanism of miR-508-3p downregulation remains unclear in ovarian cancer. Zhao et al (36) have reported that miR-508-3p downregulation in ovarian cancer is due to promoter hypermethylation, and that miR-508-3p may be a potential new biomarker for ovarian cancer. Further studies are required to investigate the deregulation mechanism. The results of the present study confirmed that miR-508-3p expression was associated with improved prognosis and was an independent predictor for OS in ovarian cancer by multivariate analysis, highlighting its potential value as a prognostic biomarker in ovarian cancer.

It has been reported that CCNA2 expression in tumors may also have prognostic value $(11,37)$. In the present study, high CCNA2 expression was associated with poor survival. Multivariate analysis revealed that CCNA2 was an independent predictor of OS in ovarian cancer. A previous study has reported that upregulation of MMP7 in serum was associated with a significantly impaired OS in cancers (31). The present data demonstrated that MMP7 expression in tumor tissues was associated with a poor survival of patients with ovarian cancer, but MMP7 was not an independent predictor for OS. The results of the present study also demonstrated that CCNA2 and MMP7 expression was negatively associated with miR-508-3p expression in tumor tissues, which suggested that miR-508-3p downregulation may contribute to the increased expression of CCNA2 and MMP7 in ovarian cancer. Thus, CCNA2 and 
MMP7 may be promising therapeutic candidates for ovarian cancer.

Taken together, the results of the present study demonstrated that $\mathrm{miR}-508-3 \mathrm{p}$ suppressed the proliferation, migration and invasion of ovarian cancer cells by directly regulating CCNA2 and MMP7 and may serve as an independent prognostic factor for the OS of patients with ovarian cancer. Thus, these results are encouraging and suggest that miR-508-3p and its downstream molecules CCNA2 and MMP7 are potential targets for the treatment of ovarian cancer.

\section{Acknowledgements}

The authors would like to thank Professor Shizhu Yu and Professor Chunsheng Kang (Department of Neuropathology, Tianjin Neurological Institute, Tianjin Medical University General Hospital) for their discussion and revision of this manuscript.

\section{Funding}

This work was supported by the National Natural Science Foundation of China (grant nos. 81772790 to FXX, 81602292 to FG and 81472761 to GYL), the Science \& Technology Foundation for Selected Overseas Chinese Scholars, Bureau of Personnel of China, Tianjin (grant no. 2016017 to FG), Tianjin Medical University General Hospital Grant (grant no. ZYYFY2014004 to FG) and the Postgraduate Innovation Fund of 13th Five-year comprehensive investment, Tianjin Medical University (grant no. YJSCX201812 to KZ).

\section{Availability of data and materials}

All data generated or analyzed during this study are available from the corresponding author on reasonable request.

\section{Authors' contributions}

FG and FXX designed the study. FG, KZ, MYL and LC performed the experiments. GYL, YY,WYT and FT performed the statistical analysis. YFZ, CG, JPG and YMW analyzed the clinical specimens. FG, YMW and FXX wrote and revised the manuscript. All authors have read and approved the final manuscript.

\section{Ethics approval and consent to participate}

This study was approved by the Ethics Committee of Tianjin Medical University General Hospital (TGH) and all procedures followed the principles of the Declaration of Helsinki. All subjects provided written informed consent prior to participation.

\section{Patient consent for publication}

Not applicable.

\section{Competing interests}

The authors declare that they have no competing interests.

\section{References}

1. Siegel RL, Miller KD and Jemal A: Cancer Statistics, 2017. CA Cancer J Clin 67: 7-30, 2017.

2. Chen W, Zheng R, Baade PD, Zhang S, Zeng H, Bray F, Jemal A Yu XQ and He J: Cancer statistics in China, 2015. CA Cancer J Clin 66: 115-132, 2016.

3. Nasioudis D, Kahn R, Chapman-Davis E, Frey MK, Caputo TA Witkin SS and Holcomb K: Impact of hospital surgical volume on complete gross resection (CGR) rates following primary debulking surgery for advanced stage epithelial ovarian carcinoma. Gynecol Oncol 154: 401-404, 2019.

4. Rojas V, Hirshfield KM, Ganesan S and Rodriguez-Rodriguez L: Molecular Characterization of Epithelial Ovarian Cancer: Implications for Diagnosis and Treatment. Int J Mol Sci 17: 17, 2016.

5. Allemani C, Weir HK, Carreira H, Harewood R, Spika D, Wang XS, Bannon F, Ahn JV, Johnson CJ, Bonaventure A, et al: CONCORD Working Group: Global surveillance of cancer survival 1995-2009: Analysis of individual data for $25,676,887$ patients from 279 population-based registries in 67 countries (CONCORD-2). Lancet 385: 977-1010, 2015.

6. Zhai Q, Zhou L, Zhao C, Wan J, Yu Z, Guo X, Qin J, Chen J and Lu R: Identification of miR-508-3p and miR-509-3p that are associated with cell invasion and migration and involved in the apoptosis of renal cell carcinoma. Biochem Biophys Res Commun 419: 621-626, 2012.

7. Yu X, Zhang X, Bi T, Ding Y, Zhao J, Wang C, Jia T, Han D, Guo G, Wang B, et al: MiRNA expression signature for potentially predicting the prognosis of ovarian serous carcinoma. Tumour Biol 34: 3501-3508, 2013.

8. Hidaka H, Seki N, Yoshino H, Yamasaki T, Yamada Y, Nohata N, Fuse M, Nakagawa $M$ and Enokida H: Tumor suppressive microRNA-1285 regulates novel molecular targets: Aberrant expression and functional significance in renal cell carcinoma. Oncotarget 3: 44-57, 2012.

9. Huang T, Kang W, Zhang B, Wu F, Dong Y, Tong JH, Yang W, Zhou Y, Zhang L, Cheng AS, et al: miR-508-3p concordantly silences NFKB1 and RELA to inactivate canonical NF- $\kappa \mathrm{B}$ signaling in gastric carcinogenesis. Mol Cancer 15: 9, 2016.

10. Yamamoto S, Tsuda H, Miyai K, Takano M, Tamai S and Matsubara O: Cumulative alterations of p27-related cell-cycle regulators in the development of endometriosis-associated ovarian clear cell adenocarcinoma. Histopathology 56: 740-749, 2010.

11. Lee YH, Heo JH, Kim TH, Kang H, Kim G, Kim J, Cho SH, An HJ: Significance of cell cycle regulatory proteins as malignant and prognostic biomarkers in ovarian epithelial tumors. Int $\mathbf{J}$ Gynecol Pathol 30: 205-217, 2011.

12. Song N, Liu H, Ma X and Zhang S: Placental growth factor promotes metastases of ovarian cancer through miR-543regulated MMP7. Cell Physiol Biochem 37: 1104-1112, 2015.

13. Yu B, Liu X and Chang H: MicroRNA-143 inhibits colorectal cancer cell proliferation by targeting MMP7. Minerva Med 108: 13-19, 2017.

14. Berek JS, Crum C and Friedlander M: Cancer of the ovary, fallopian tube, and peritoneum. Int J Gynaecol Obstet 119 (Suppl 2): S118-S129, 2012.

15. Yang D, Sun Y, Hu L, Zheng H, Ji P, Pecot CV, Zhao Y, Reynolds S, Cheng H, Rupaimoole R, et al: Integrated analyses identify a master microRNA regulatory network for the mesenchymal subtype in serous ovarian cancer. Cancer Cell 23: 186-199, 2013.

16. Parker BC, Annala MJ, Cogdell DE, Granberg KJ, Sun Y, Ji P, Li X, Gumin J, Zheng H, Hu L, et al: The tumorigenic FGFR3-TACC3 gene fusion escapes miR-99a regulation in glioblastoma. J Clin Invest 123: 855-865, 2013.

17. Zarkowska T, U S, Harlow E and Mittnacht S: Monoclonal antibodies specific for underphosphorylated retinoblastoma protein identify a cell cycle regulated phosphorylation site targeted by CDKs. Oncogene 14: 249-254, 1997.

18. Wang X, Hu Y, Cui J, Zhou Y and Chen L: Coordinated targeting of MMP-2/MMP-9 by miR-296-3p/FOXCUT exerts tumor-suppressing effects in choroidal malignant melanoma. Mol Cell Biochem 445: 25-33, 2018.

19. Sun Y, Hu L, Zheng H, Bagnoli M, Guo Y, Rupaimoole R, Rodriguez-Aguayo C, Lopez-Berestein G, Ji P, Chen K, et al: miR-506 inhibits multiple targets in the epithelial-to-mesenchymal transition network and is associated with good prognosis in epithelial ovarian cancer. J Pathol 235: 25-36, 2015. 
20. Liu G, Sun Y, Ji P, Li X, Cogdell D, Yang D, Parker Kerrigan BC, Shmulevich I, Chen K, Sood AK, et al: miR-506 suppresses proliferation and induces senescence by directly targeting the CDK4/6-FOXM1 axis in ovarian cancer. J Pathol 233: 308-318, 2014.

21. Sun Y, Guo F, Bagnoli M, Xue FX, Sun BC, Shmulevich I, Mezzanzanica D, Chen KX, Sood AK, Yang D, et al: Key nodes of a microRNA network associated with the integrated mesenchymal subtype of high-grade serous ovarian cancer. Chin J Cancer 34: 28-40, 2015.

22. Lin C, Liu A, Zhu J, Zhang X, Wu G, Ren P, Wu J, Li M, Li J and Song L: miR-508 sustains phosphoinositide signalling and promotes aggressive phenotype of oesophageal squamous cell carcinoma. Nat Commun 5: 4620, 2014.

23. Cribier A, Descours B, Valadão AL, Laguette N and Benkirane $\mathrm{M}$ : Phosphorylation of SAMHD1 by cyclin A2/CDK1 regulates its restriction activity toward HIV-1. Cell Rep 3: 1036-1043, 2013.

24. Das E, Jana NR and Bhattacharyya NP: MicroRNA-124 targets CCNA2 and regulates cell cycle in STHdh(Q111)/Hdh(Q111) cells. Biochem Biophys Res Commun 437: 217-224, 2013

25. van Olphen SH, Ten Kate FJ, Doukas M, Kastelein F, Steyerberg EW, Stoop HA, Spaander MC, Looijenga LH, Bruno MJ and Biermann K: ProBar-Study Group: Value of cyclin A immunohistochemistry for cancer risk stratification in Barrett esophagus surveillance: A multicenter case-control study. Medicine (Baltimore) 95: e5402, 2016.

26. Cooper WA, Kohonen-Corish MR, McCaughan B, Kennedy C, Sutherland RL and Lee CS: Expression and prognostic significance of cyclin B1 and cyclin A in non-small cell lung cancer. Histopathology 55: 28-36, 2009.

27. Nozoe T, Inutsuka S, Honda M, Ezaki T and Korenaga D: Clinicopathologic significance of cyclin A expression in colorectal carcinoma. J Exp Clin Cancer Res 23: 127-133, 2004.

28. Gopinathan L, Tan SL, Padmakumar VC, Coppola V, Tessarollo L and Kaldis P: Loss of Cdk2 and cyclin A2 impairs cell proliferation and tumorigenesis. Cancer Res 74: 3870-3879, 2014.

29. Srethapakdi M, Liu F, Tavorath R and Rosen N: Inhibition of Hsp90 function by ansamycins causes retinoblastoma gene product-dependent G1 arrest. Cancer Res 60: 3940-3946, 2000.
30. Xu J, e C, Yao Y, Ren S, Wang G and Jin H: Matrix metalloproteinase expression and molecular interaction network analysis in gastric cancer. Oncol Lett 12: 2403-2408, 2016.

31. Klupp F, Neumann L, Kahlert C, Diers J, Halama N, Franz C, Schmidt T, Koch M, Weitz J, Schneider M, et al: Serum MMP7, MMP10 and MMP12 level as negative prognostic markers in colon cancer patients. BMC Cancer 16: 494, 2016.

32. Zhu R and Tian Y: Astrocyte elevated gene-1 increases invasiveness of NSCLC through up-regulating MMP7. Cell Physiol Biochem 37: 1187-1195, 2015.

33. Maurel J, Nadal C, Garcia-Albeniz X, Gallego R, Carcereny E, Almendro V, Mármol M, Gallardo E, Maria Augé J, Longarón R, et al: Serum matrix metalloproteinase 7 levels identifies poor prognosis advanced colorectal cancer patients. Int J Cancer 121: 1066-1071, 2007.

34. Niedworok C, Tschirdewahn S, Reis H, Lehmann N, Szucs M, Nyirady P, Romics I, Rubben H and Szarvas T: serum chromogranin a as a complementary marker for the prediction of prostate cancer-specific survival. Pathol Oncol Res 23: 643-650, 2017.

35. Zhang Q, Liu S, Parajuli KR, Zhang W, Zhang K, Mo Z, Liu J, Chen Z, Yang S, Wang AR, et al: Interleukin-17 promotes prostate cancer via MMP7-induced epithelial-to-mesenchymal transition. Oncogene 36: 687-699, 2017.

36. Zhao L, Wang W, Xu L, Yi T, Zhao X, Wei Y, Vermeulen L, Goel A, Zhou S and Wang X: Integrative network biology analysis identifies miR-508-3p as the determinant for the mesenchymal identity and a strong prognostic biomarker of ovarian cancer. Oncogene 38: 2305-2319, 2019.

37. Li HP, Ji JF, Hou KY, Lei YT, Zhao HM, Wang J, Zheng J, Liu JY, Wang MP, Xiao Y, et al: Prediction of recurrence risk in early breast cancer using human epidermal growth factor 2 and cyclin A2. Chin Med J (Engl) 123: 431-437, 2010.

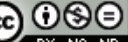

This work is licensed under a Creative Commons Attribution-NonCommercial-NoDerivatives 4.0 International (CC BY-NC-ND 4.0) License. 